\title{
Interference grating structures in photonic crystal circuits
}

\author{
O. O. Karakilinc · M. S. Dinleyici
}

Received: 14 March 2011 / Accepted: 11 August 2011 / Published online: 31 August 2011

(C) Springer Science+Business Media, LLC. 2011

\begin{abstract}
Photonic Crystal Circuits have been widely investigated for various optoelectronics device applications. And the gratings in photonics devices are indispensable tool, which is very common for light manipulation. In this study, an interference grating structure formed by Gaussian beam interferences in photonic crystal waveguide such that having Kerr type nonlinearity is proposed and its transmission characteristics are investigated. FiniteDifference Time-Domain method is used to analyze the device characteristics. According to simulation results, the interference grating has shown special transmission characteristics that can bring tunability for photonic crystal devices. This can be an effective method for controlling optical signals in the photonic crystal for all optical switching/routing applications as a part of add/drop multiplexing.
\end{abstract}

Keywords Photonic crystal circuits $\cdot$ Kerr nonlinearity $\cdot$ Beam interference $\cdot$ Optical switching

\section{Introduction}

Photonic crystals (PhCs) are artificial materials which include a periodic arrangement of dielectric medium in one, two or three dimensions. One of the most important properties of PhCs is photonic bandgap (PBG). The formation of PBG is similar to the formation of the energy bandgap in the semiconductor crystals (Joannopoulos et al. 2008). PhCs exhibit photonic band gaps depending on their geometry and refractive index profiles, many forms of these structures have been proposed and investigated in literature (Joannopoulos et al. 2008;

O. O. Karakilinc $(\varangle)$

Department of Electrical and Electronics Engineering, Pamukkale University, 20070 Denizli, Turkey e-mail: okarakilinc@pau.edu.tr

M. S. Dinleyici

Department of Electrical and Electronics Engineering, Izmir Institute of Technology, 35437 Urla, Izmir, Turkey

e-mail: salihdinleyici@iyte.edu.tr 
Mingaleev and Kivshar 2002; McGurn 2000). One example for refractive index profile is the gratings that may be permanently or temporarily created by interferences of two or more beams. By creating the gratings into $\mathrm{PhCs}$, one can control and manipulate the light, i.e., optical signal (Chen et al. 2007; Akmansoy et al. 2008; Liu et al. 2009; Vigneron and Lousse 2007).

In PhCs, optical light signals can be confined into a narrower region than the conventional optical waveguides, which is very attractive for enhancement of the nonlinear effects. Exploiting this feature of the $\mathrm{PhCs}$, a Kerr type nonlinear waveguide as an interference grating medium can be embedded and the nonlinearity can conveniently be available for tuning applications. Hence, the optimization of optical and physical parameters of the structures is necessary to accurately design photonic band gap based devices. In recent years a lot of researches have been carried out for finding $\mathrm{PhC}$ structures that exhibit various functions (filters, couplers, multiplexers) necessary in the fields of photonics, optical communications etc. (Selim et al. 2011; Djavid and Abrishamian 2007). Furthermore, integration of these grating devices is another research area that has to be deeply investigated. Subsequently, temporal/dynamical manipulation of the $\mathrm{PhCs}$ come to play in order to switch the light/optical signal between the waveguide channels or may be among the layers. Due to very fast response requirement of optical communications, the most suitable approach to control bandgap and then realization of fast optical devices is to exploit nonlinearity in PhCs. Nonlinearity in $\mathrm{PhC}$ can be exploited either by applied external electric field which cause the refractive index change uniformly or interference of multiple beams which makes refractive index modulation periodical. Nonlinear photonic crystal with periodic modulation of second order $\left(\chi^{(2)}\right)$ and third order $\left(\chi^{(3)}\right)$ nonlinear susceptibility have drawn wide attention in the literature both theoretically and experimentally (Mingaleev and Kivshar 2002; Berger 1998). Because of the very short relaxation time (Bonnefois et al. 2009) and fast response time about $10^{-15} \mathrm{~s}$ (Boyd 2008), Kerr type nonlinearity has been pioneered for the realizing optical fast switching application in the recent years (Liu et al. 2009; Katouf et al. 2005).

On the other hand, recent progresses in $\mathrm{PhC}$ studies have evolved into 3D structures in which the propagating light needs to be routed/switched among the layers or the light paths in the layer. That configurations in general, called PhC Circuits (PCC), may also include functions more than just bending or coupling (Ren et al. 2008), but rather dynamically controlling and modulating the light signal in order to switching and/or routing among the layers or the light paths.

Assembling multi function photonic components into $\mathrm{PhC}$ structure has drawn very much attention among the researchers in that field for the last a few years (Grillet et al. 2010). The gratings in photonics are indispensable common tools for light manipulations for a long time, and it can also be employed in PCCs for the similar functions. The gratings can be apodized and/or chirped which the amplitude and the period along its direction are not uniform. Until now, chirped and/or apodized gratings have been applied for modulation and/or switching/routing in optical communication systems (Chen et al. 2010; Yesayan and Piche 2005; Goncharenko and Zervas 2002). In PCCs the apodized gratings have also been exploited for focusing etc. (Liu et al. 2009; Kurt et al. 2008; Yokoi et al. 2006) by means of modifying the period, the radius or dielectric constant of the dielectric rods. Following up the idea, the gratings may be useful tool in PCC, too, to design advanced photonic structures for complex functions of compound all-optical devices.

In this paper, applications of the interference grating structures in PCCs have been investigated. First of all, intensity distribution for interference of two Gaussian beams in Kerr type nonlinear medium is obtained, and then this interference equation is taken as a grating function in PCC. Transmission characteristics of that transient grating nonlinear waveguide have 
been analyzed by simulating the model using FDTD method. The results for transmission characteristics have been presented for optimization parameters of Kerr nonlinear coefficients and beam incidence angles. Also, the characteristics are extended for two wavelengths for spectral performance evaluation.

\section{Interference of Gaussian beams}

When two or more coherent optical waves are present simultaneously in the same region of space, the waves interfere and generate a periodic intensity distribution (Boor et al. 2009). For two or more coherent beams (instead of waves) the intensity distribution will be spatially quasi-periodic and non-uniform (Li et al. 1997). For simplicity, in this work, two-dimensional Gaussian beams interference geometry (two beams are in the same plane) has been considered and one dimensional interference grating geometry is depicted in the Fig. 1.

After the coordinate transformation and the first order approximation, complex Gaussian beam equation can be written as follows (Akin 2005);

$$
\begin{aligned}
U(x, y)= & \frac{A}{\left(L+y \cdot \cos (\theta)-x \cdot \sin (\theta)+i \cdot z_{0}\right)} \\
& \times \exp [-i k(L+y \cdot \cos (\theta)-x \cdot \sin (\theta))] \\
& \times \exp \left[\frac{-1}{\left.2\left[L+y \cdot \cos (\theta)-x \cdot \sin (\theta)+i \cdot z_{0}\right)\right]} \cdot i k\left[(x \cdot \cos (\theta)+y \cdot \sin (\theta))^{2}\right]\right]
\end{aligned}
$$

Where $z_{0}$ is Rayleigh range, $\theta$ is incidence angle, $L$ is the axial distance from minimum waist of beam to interference plane and $A$ is constant.

Interference equation will be in the following equation.

$$
I(x, y, t)=\left|U_{1}(x, y, t)+U_{2}(x, y, t)\right|^{2}
$$

The period of the uniform grating $\Lambda$ is given as

$$
\Lambda=\frac{\lambda_{\text {beam }}}{2 \sin \theta}
$$

where $\lambda_{\text {beam }}$ is the wavelength of the Gaussian beam and $\theta$ is the incident angle of beam.

Fig. 1 Geometry of interference of Gaussian beams

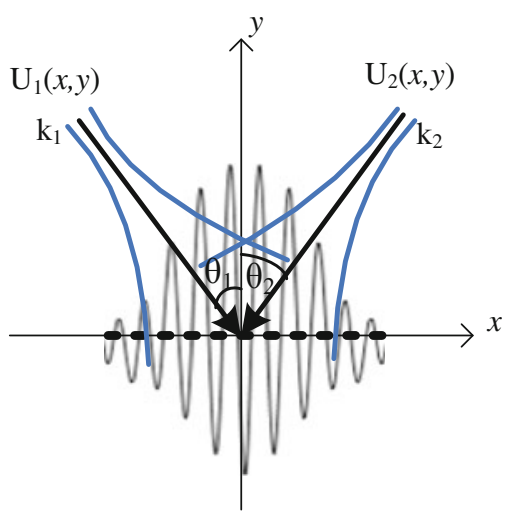


Bragg wavelength which corresponds to input signal reflected back in a uniform grating structure is given as,

$$
\lambda_{B}=2 \Lambda \bar{n}
$$

where $\bar{n}$ the effective average refractive index as defined in Saleh and Teich (2007).

In general, an interference grating structure can be generated in a third order (Kerr type) nonlinear waveguide by exposing the intensity distribution of the interference. The nonlinear Kerr medium will be modified according to refractive index profile of the intensity distribution, because the Kerr type nonlinearity is a linear function of the optical intensity $I$ in the following equations,

$$
n(I)=n+n_{2} I
$$

and $n_{2}$ is given as

$$
n_{2}=\frac{3 \eta_{0}}{n^{2} \varepsilon_{0}} \chi^{(3)}
$$

where $n$ is refractive index, $\Delta n=n_{2} I$ is nonlinear index change, $\eta_{0}$ is impedance of free space, and $\chi^{(3)}$ is Kerr coefficient (Saleh and Teich 2007).

When the beams interference occurs at the $y=0$ plane, a stationary grating pattern is created. The grating period, along the $x$-direction, depends on the interference beam angle, the writing beam wavelength, and the interference plane $(y)$.

According to the interference Eq. (2), there are two terms that make the refractive index profile different than the uniform grating. The first term is an amplitude modulation (envelope) that diminishes exponentially and the second term is a pitch modulation that modifies the grating period. The grating length is bounded with the envelope function of the interference equation (Akin and Dinleyici 2010).

\section{Computation and simulation method}

For the simulation, $U_{1}$ and $U_{2}$ Gaussian beams in interference equation are taken identical and applied to Kerr type nonlinear waveguide in $\mathrm{PhC}$ simultaneously. The way to deliver the interfering beams into the nonlinear waveguide is through the bulk of PhC. Propagation modes are obtained from bulk $\mathrm{PhC}$ band structure graphs thus the possible interference angles of the beam are delimited. Propagated beam frequency $0.949 c / a$ is chosen from the bulk $\mathrm{PhC}$ band structure as shown in the Fig. 2. Thereby, propagation of interfering beams through bulk $\mathrm{PhC}$ is guaranteed.

Interference of beams and corresponding electric field profile in interference region versus the different incident angle are pictured in the following simulation graphics (Figs. 3, 4).

It is expected to incremental refractive index change of the medium in accordance with interference equation depending on optical property of the material. As simulation geometry depicted in Fig. 5, there is a hexagonal photonic crystal structure with air holes into linear polymer substrate with nonlinear Kerr type line defect waveguide that placed at the center part. The waveguide embedded into the $\mathrm{PhC}$ is a nonlinear polymer channel waveguide. Nonlinear interaction in surrounding materials is weak and can be neglected.

The radius of the rods is chosen $0.2 a$ and the refractive index of background Silicon Dioxide (SiO2) and the nonlinear material, Methyl Red (MR) doped Polyvinyl Alcohol (PVA) are 1.46 and 1.488 respectively. The lattice constant ' $a$ ' is in unit of $\mu \mathrm{m}$ and all the values in the simulation are normalized with $\mu \mathrm{m}$. Also, frequency is normalized to $a / \lambda$. When the 

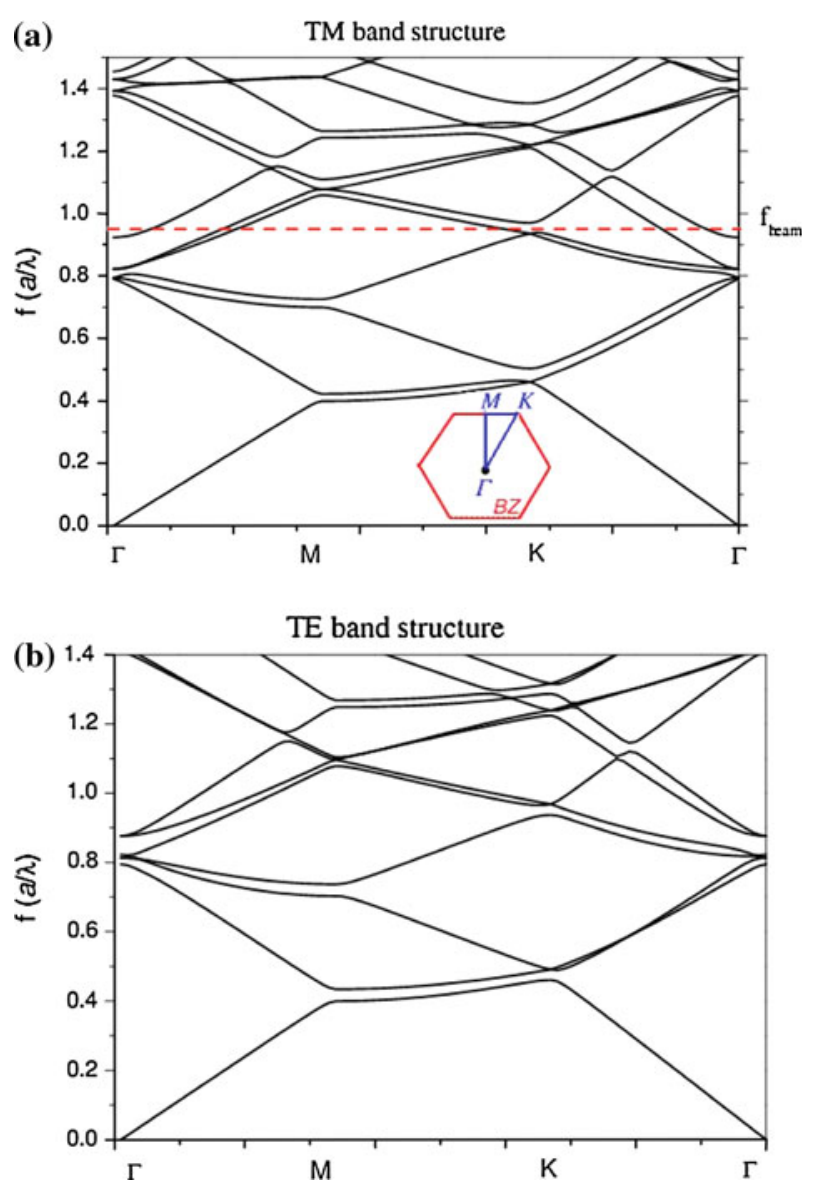

Fig. 2 a TM and $\mathbf{b}$ TE band structures

lattice constant $a$ is taken $1 \mu \mathrm{m}$, the normalized frequency 0.65 is wavelength of $1.54 \mu \mathrm{m}$ which is in the communication window. Also, for the given geometry, the waveguide width $3 a \sqrt{3} / 2$ becomes $2.59 \mu \mathrm{m}$ if $a$ is taken $1 \mu \mathrm{m}$, therefore this correspondence to single mode waveguide for the given frequency range.

Methyl Red (MR) doped Polyvinyl Alcohol (PVA) is chosen as nonlinear waveguide material because of the high nonlinear coefficient, high chemical stability and suitability for the sub micron scale fabrication process The nonlinearity considered in this study is instantaneous nonlinearity and third order nonlinear coefficient of doped PVA can be taken as $2 \times 10^{-9} \mathrm{~cm}^{2} / \mathrm{W}$ compatible with the literature (Xiaoqiang et al. 2010; Tingchao et al. 2007).

There are a number of methods in the literature for simulating the nonlinear optical $\mathrm{PhC}$ structures (Maksymov et al. 2006; Taflove and Hagness 2005; Reinke et al. 2006; Kamalakis and Sphicopoulos 2007). FDTD method is the most useful method that is used for analyzing nonlinear optical devices, which are composed of PhCs and nonlinear waveguides. The simulation results are obtained with MEEP which is a free finite-difference time-domain (FDTD) simulation software package developed at MIT photonic research group. MEEP has ability to 
(a)

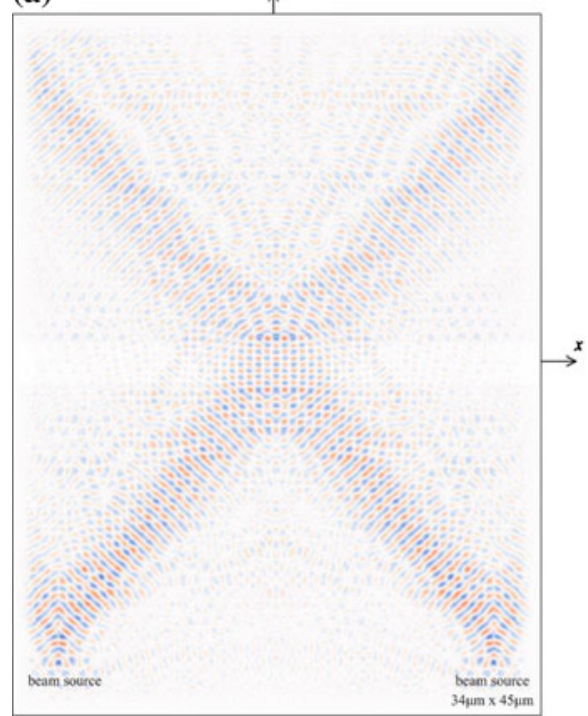

(b) $\uparrow^{y}$

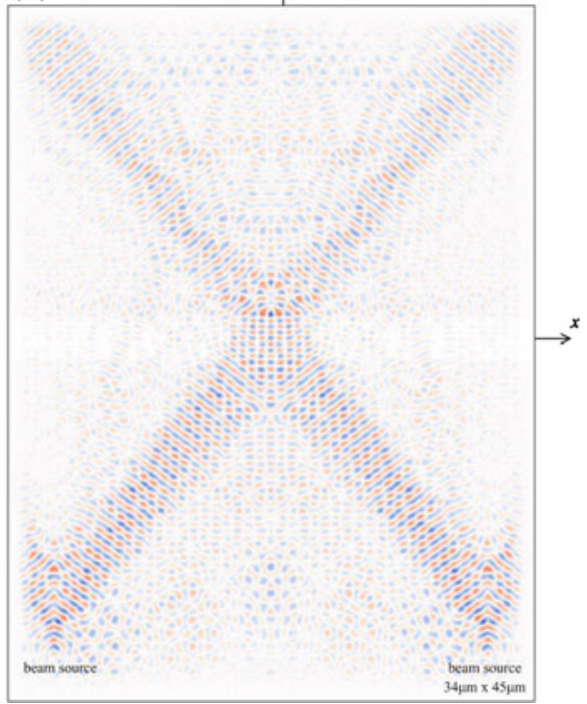

Fig. 3 Gaussian beams interference pattern in $\mathrm{PhC}$ structure $\left(U_{1}, U_{2}\right.$ are identical and incident angle is taken a $\theta=45^{\circ} \mathbf{b} \theta=50^{\circ}$ )

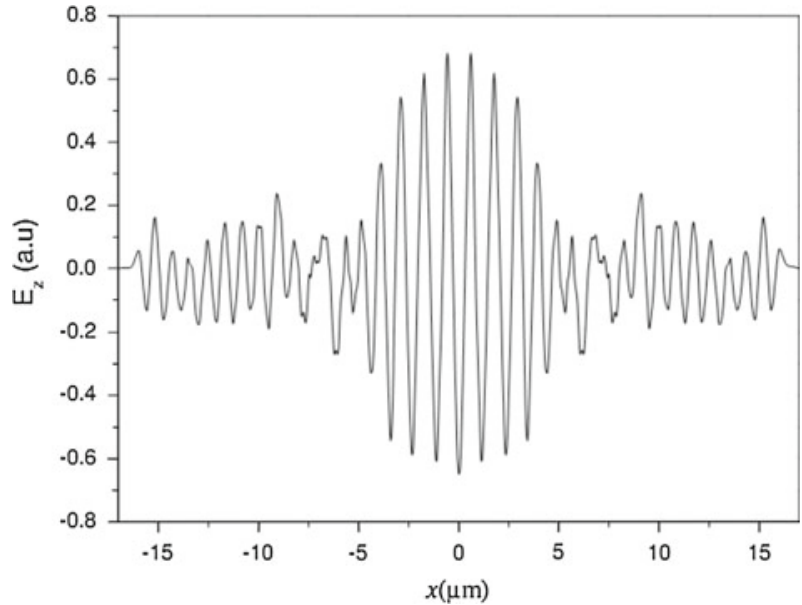

Fig. 4 Electric field variation along the grating axis in $\mathrm{PhC}$ nonlinear waveguide belong to Fig. 2a

simulate the third order instantaneous isotropic nonlinear photonic structures (Oskooi et al. 2010).

\section{Simulation results}

Transmission characteristics and relation with some beam parameters have been investigated and presented in this section. Input signal source is defined in the waveguide as a carrier signal. Propagated beam frequency $0.949 c / a$ is chosen from the bulk $\mathrm{PhC}$ band structure as 


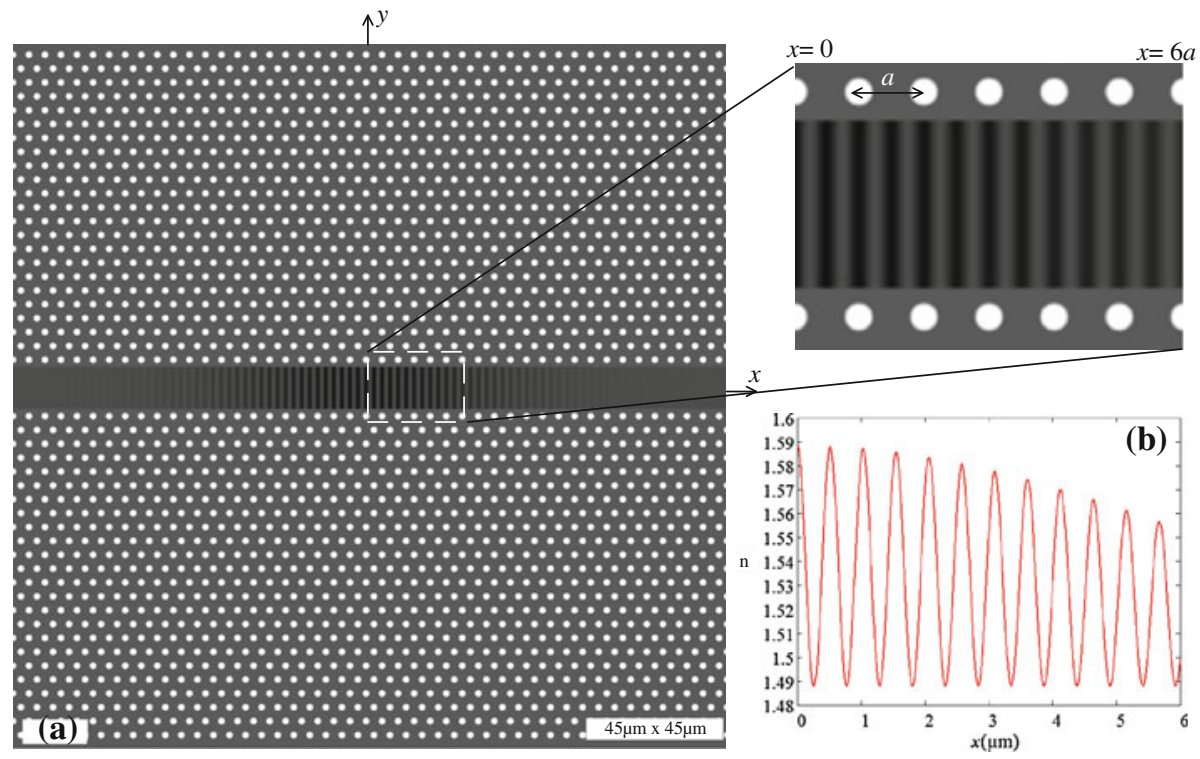

Fig. 5 a Simulation structure and $\mathbf{b}$ index profile of grating

shown in the Fig. 2. Throughout the simulations, all beams are TE polarized (TM, according to MEEP).

Transmission coefficient of the interference grating is defined as the ratio of the output flux at the end of the waveguide to the output flux measured after the interference grating applied. Transmission characteristics of nonlinear waveguide versus to beam parameters like $\theta$ (incident angle), $\lambda$ (control beam wavelength), $P_{0}$ (beam power) are given in the following subsections.

\subsection{Transmission characteristics versus $\theta$}

The transmission coefficients are obtained for the interference beam angle of incidence $45^{\circ}$ and $50^{\circ}$. Corresponded beam frequency is $0.949 \mathrm{c} / \mathrm{a}$ and chosen from dispersion diagram in Fig. 2. The interfering beam wavelength and the power, consequently nonlinear index deviation, are held constant as $1.053 \mu \mathrm{m}$ and $P_{0}$ respectively. The power $\left(P_{0}\right)$ required to make necessary refractive index change of 0.1 is about $0.5 \mathrm{~W} / \mu \mathrm{m}^{2}$.

As the results shown in the Fig. 6, when interference angle of incidence is increased, the stop frequency shifted to the higher frequency region and the bandwidth ( $3 \mathrm{~dB}$ bandwidth) also increases. The bandwidth is about $0.022 c / a$ at $45^{\circ}$ which is about $6.6 \mathrm{THz}$ if the lattice constant is taken $1 \mu \mathrm{m}$. This kind of characteristic is predicted and confirmed by the Bragg law given in the Eqs. (3) and (4). When the angle of incidence is increased, the grating period $(\Lambda)$ decreases; corresponding Bragg wavelength $\left(\lambda_{B}\right)$ decreases too. On the other hand, grating length increases for the larger incidence angle. Hence, the contrast ratio reaches $10 \mathrm{~dB}$ at the higher frequencies rather than $8 \mathrm{~dB}$ at the lower part of the spectrum with the change of incidence angle. 


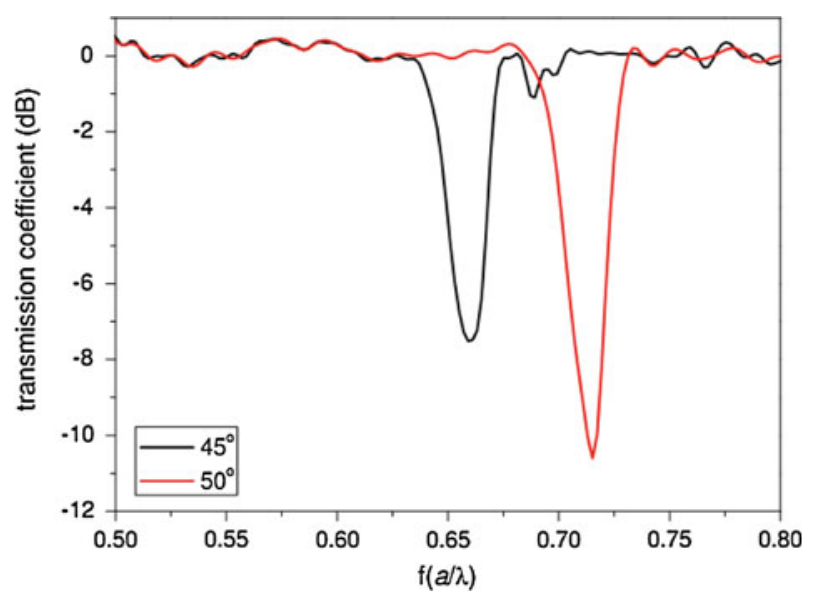

Fig. 6 Transmission coefficient for two different $\theta$ values $\left(P=P_{0}\right.$ and $\left.\lambda=1.053 \mu \mathrm{m}\right)$

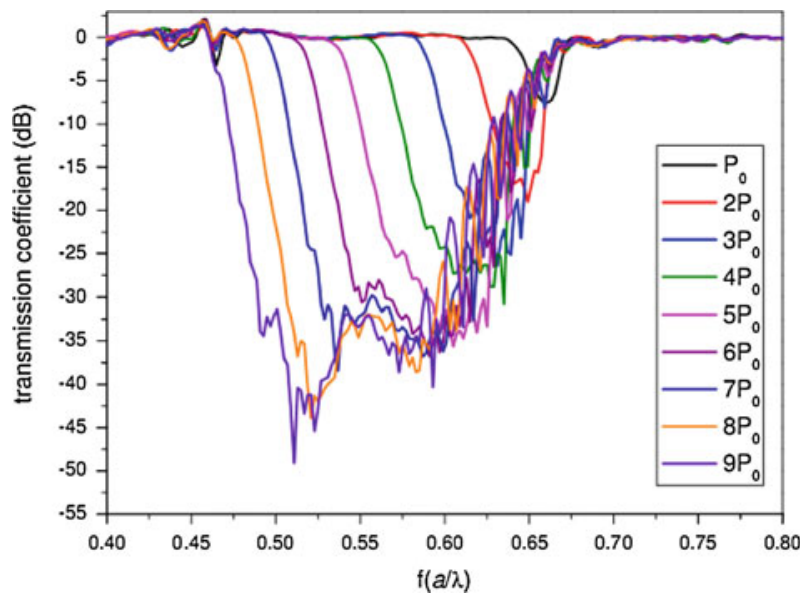

Fig. 7 Transmission coefficient for power variation $\left(\theta=45^{\circ}\right.$ and $\left.\lambda=1.053 \mu \mathrm{m}\right)$

\subsection{Transmission characteristics versus $\left(P_{0}\right)$}

The effect of beams power on the stop frequency is presented in the following Figs. 7 and 8. Power density still remains under the threshold damage power level of MR PVA polymer (Tingchao et al. 2007). Actually, this power is belong to two beam interferences in the center of grating region. Power of one beam is $0.125 \mathrm{~W} / \mu \mathrm{m}^{2}$ for $P_{0}$.

When the beam power is increased and then the refractive index is modified accordingly. Hence, the bandwidth is broadened and the stop frequency is shifted to lower frequency part of the spectrum. That can be explained with an increase in the effective average refractive index; therefore, the Bragg wavelength $\left(\lambda_{B}\right)$ shifts according to the Bragg law in Eq. (4). Noticeably, the contrast ratio (Notomi et al. 2005) enhances from 8 to $45 \mathrm{~dB}$ with the increased $P_{0}$. The calculated crosstalk between adjacent frequencies is significantly low with the increased power change above $2 P_{0}$ where $P_{0}$ is the power requirements for $\Delta n \sim 0.1$. Interestingly, the beam power change dominantly affects the bandwidth as shown in the Fig. 8, in which 


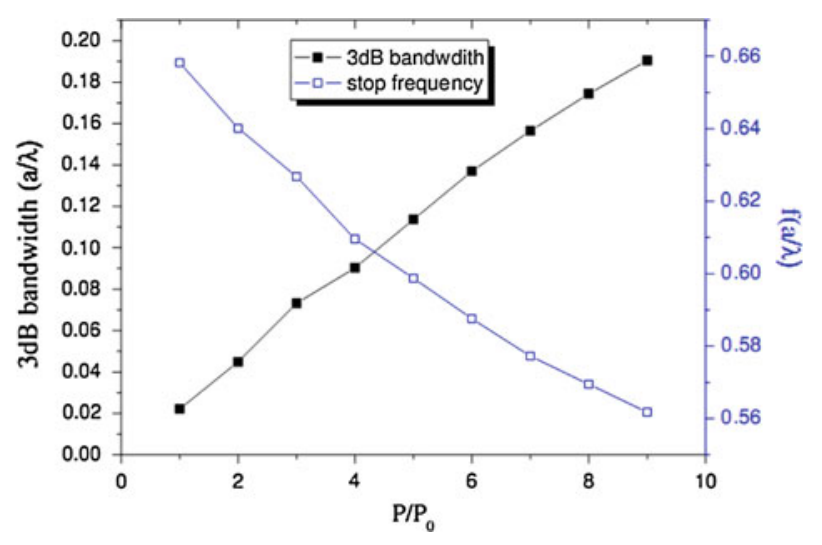

Fig. 8 Shifting of stop frequency and $3 \mathrm{~dB}$ bandwidth for various beam power

the bandwidth broadens to $0.190 c / a$ (57 Thz for $a$ is $1 \mu \mathrm{m}$ ) Therefore, that feature can be used in applications that require ultra-wide-band width; since it can easily be tuned with the beam power.

\subsection{Transmission characteristics versus $\lambda_{\text {beam }}$}

The effect of interfering beam frequency on the transmission characteristic is considered for two wavelengths $\lambda_{1}=1 \mu \mathrm{m}, \lambda_{2}=1.053 \mu \mathrm{m}$. The beam angle of incidence and the amplitude are held constant. According to the Eqs. (3) and (4), when the beam wavelength is increased, Bragg period $(\Lambda)$, corresponding Bragg wavelength $\left(\lambda_{B}\right)$ and grating length are increased. When the wavelength is decreased, the stop frequency shifts toward to higher frequency region of the spectrum. For these wavelengths, the calculated crosstalk is $-8 \mathrm{~dB}$.

At the end of the study, Fig. 9 is confirmed with the FDTD simulation. As we can see in Fig. 9, there is a stop frequency at $0.65 c / a$ for the control beam of $1.053 \mu \mathrm{m}$. In order

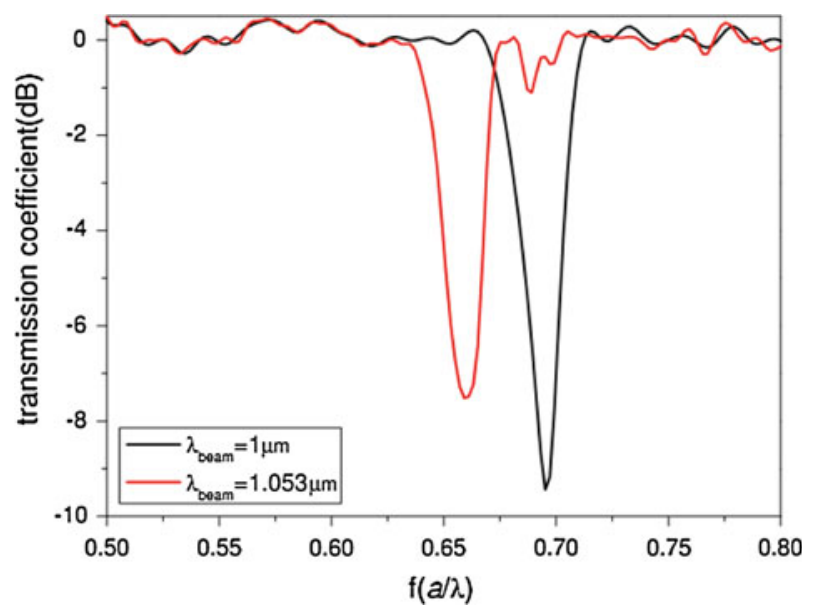

Fig. 9 Transmission coefficient for beam wavelength $\left(\theta=45^{\circ}\right.$ and $\left.P=P_{0}\right)$ 

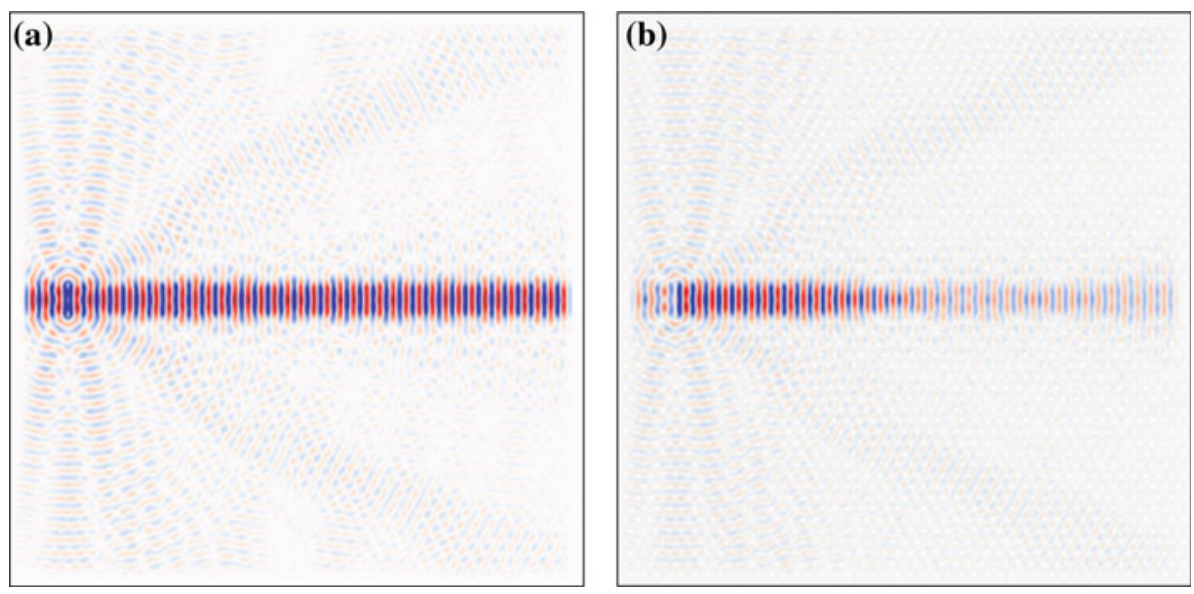

Fig. 10 Input signal propagation a without grating $\left(f_{\text {input }}=0.65\right)$. b with grating $\left(f_{\text {input }}=0.65, \lambda=\right.$ $1.053 \mu \mathrm{m}, \theta=45^{\circ}$ and $P=P_{0}$ )

to demonstrate the stop mechanism, continuous wave input signal source is chosen $0.65 c / a$ and launched into the waveguide as carrier signal.

In the Fig. 10, the propagation of input signal is shown with/without the grating. Under the grating condition, the input signal is literally stopped as in agreement with the simulation in Fig. 9. The transmission is down to $-8 \mathrm{~dB}$.

\section{Conclusions}

The concept of interference grating structures formed by Interference of Gaussian beams in a nonlinear $\mathrm{PhC}$ waveguide medium is proposed and investigated. These gratings have profiles that chirped and apodized. An embedded Kerr type nonlinear waveguide in the PhCs has been first time considered as the beam interference grating medium for manipulating the optical signals as far as we know. Finite-Difference Time-Domain (FDTD) Method is used to analyze these type photonic structures for the transmission characteristics.

Since the interference grating structures are slightly chirped and apodized, in the transmission characteristics stop frequency with various bandwidths has been observed in the Figs. 6, 7, and 9. In the Figs. 6 and 7 the contrast ratio is in the range of 8 to $45 \mathrm{~dB}$ and becomes lower in the long wavelength region of the spectrum.

Importantly, the stop frequency can be detuned with the interference angle about $0.05 \mathrm{c} / \mathrm{a}$ (15 THz if $a$ is $1 \mu \mathrm{m}$ ) when the angle is inclined $5^{\circ}$. Similarly, the stop frequency can also be detuned about $0.015 c / a(4.5 \mathrm{THz}$ if $a$ is $1 \mu \mathrm{m})$ when the beam power is $P \sim P_{0}$. On the other hand, the bandwidth is also affected, starting from 0.02 to $0.19 c / a$ with the frequencies ranging from 0.66 to $0.56 c / a$ for the power detuning. The power $\left(P_{0}\right)$ required to make necessary refractive index change of 0.1 is about $0.5 \mathrm{~W} / \mu \mathrm{m}^{2}$. Although, that power intensity might be achievable, the interfering beams can be confined into the narrower region by designing novel PhCs structures which accommodate the desired angle of incidence. Finally, at two beam wavelengths, 1 and $1.053 \mu \mathrm{m}$, the transmission characteristics have the stop at the frequencies 0.65 and $0.69 c / a$, with the crosstalk about $-8 \mathrm{~dB}$.

The results suggest that the interference gratings can be employed in the $\mathrm{PhC}$ circuits that require tunability for the complex photonics functions of DWDM switching/routing devices. 
Acknowledgments This work was partly supported by The Scientific and Technological Research Council of Turkey (TUBITAK), under the project 109E240.

\section{References}

Akin, O., Dinleyici, M.: An all-optical switching based on resonance breaking with a transient grating. J. Lightwave Technol. 28, 3470-3477 (2010)

Akin, O.: All optical switching via diffraction grating formed by interference of Gaussian beam. Master Thesis, pp. 42-46. Izmir Institute of High Technology (2005)

Akmansoy, E., Centeno, E., Vynck, K., Cassagne, D., Lourtioz, J.: Graded photonic crystals curve the flow of light: an experimental demonstration by the mirage effect. Appl. Phys. Lett. 92, 133501 (2008)

Berger, V.: Nonlinear photonic crystals. Phys. Rev. Lett. 81, 4136-4139 (1998)

Bonnefois, J., Guida, G., Priou, A.: Nonlinear response of a 2D photonic crystal made of Kerr effect nonlinear parallel rods of large diameter. J. Mod. Opt. 56, 646-652 (2009)

Boyd, R.W.: Nonlinear Optics. 3rd edn. Academic Press, New York (2008)

Chen, C., Li, X., Xu, K., Wu, J., Lin, J.: Photonic crystal waveguide sampled gratings. Opt. Commun. 276, 237241 (2007)

Chen, X., Li, C., Tsang, H.: Two dimensional silicon waveguide chirped grating couplers for vertical optical fibers. Opt. Commun. 283, 2146-2149 (2010)

de Boor, J., Geyer, N., Gösele, U., Schmidt, V.: Three-beam interference lithography: upgrading a Lloyd's interferometer for single-exposure hexagonal patterning. Opt. Lett. 34, 1783-1785 (2009)

Djavid, M., Abrishamian, M.S.: Photonic crystal channel drop filters with mirror cavities. Opt. Quant. Electron. 39, 1183-1190 (2007)

Goncharenko, I.A., Zervas, M.N.: Analysis method for apodised grating structures. Opt. Quant. Electron. 34, 471-479 (2002)

Grillet, C., Monat, C., Smith, C., Lee, M., Tomljenovic-Hanic, S., Karnutsch, C., Eggleton, B.: Reconfigurable photonic crystal circuits. Laser Photon. Rev. 4, 192-204 (2010)

Joannopoulos, J.D., Johnson, S.G., Winn, J.N., Meade, R.D.: Photonic Crystals: Molding the Flow of Light. Princeton University Press, New Jersey (2008)

Kamalakis, T., Sphicopoulos, T.: A new formulation of coupled propagation equations in periodic nanophotonic waveguides for the treatment of Kerr-induced, nonlinearities. IEEE J. Quant. Electron. 43, 923933 (2007)

Katouf, R., Komikado, T., Itoh, M., Yatagai, T., Umegaki, S.: Ultra-fast optical switches using 1D polymeric photonic crystals. Photon. Nanostrct. Fundam. Appl. 3, 116-119 (2005)

Kurt, H., Colak, E., Cakmak, O., Caglayan, H., Ozbay, E.: The focusing effect of graded index photonic crystals. Appl. Phys. Lett. 93, 171108 (2008)

Li, E., Tieu, K., Mackenzie, M.: Interference patterns of two focused Gaussian beams in an LDA measuring volume. Opt. Lasers Eng. 27, 395-407 (1997)

Lin, S., Huang, T., Sun, J., Wu, T.: Gradient-index phononic crystals. Phys. Rev. B. 79, 094302 (2009)

Liu, Y., Qin, F., Wei, Z., Meng, Q., Zhang, D., Li, Z.: 10 fs ultrafast all-optical switching in polystyrene nonlinear photonic crystals. Appl. Phys. Lett. 95, 131116 (2009)

Maksymov, I.S., Marsal, L.F., Pallarès, J.: An FDTD analysis of nonlinear photonic crystal waveguides. Opt. Quant. Electron. 38, 149-160 (2006)

McGurn, A.: Photonic crystal circuits: a theory for two- and three-dimensional networks. Phys. Rev. B. 61, 13235-13249 (2000)

Mingaleev, S., Kivshar, Y.: Nonlinear photonic crystals toward all-optical technologies. Opt. Photon. News 13, 48-51 (2002)

Notomi, M., Shinya, A., Mitsugi, S., Kira, G., Kuramochi, E., Tanabe, T.: Optical bistable switching action of Si high-Q photonic-crystal nanocavities. Opt. Express 13, 2678-2687 (2005)

Oskooi, A., Roundy, D., Ibanescu, M., Bermel, P., Joannopoulos, J., Johnson, S.: MEEP: a flexible free-software package for electromagnetic simulations by the FDTD method. Comput. Phys. Commun. 181, 687$702(2010)$

Reinke, C., Jafarpour, A., Momeni, B., Soltani, M., Khorasani, S., Adibi, A., Xu, Y., Lee, R.: Nonlinear finitedifference time-domain method for the simulation of anisotropic, chi((2)), and chi((3)) optical effects. J. Lightwave Technol. 24, 624-634 (2006)

Ren, H., Hu, W., Jin, Y., Jiang, C.: Design of reconfigurable optical add/drop multiplexer based on two-dimensional photonic crystals. Opt. Eng. 47, 123001 (2008)

Saleh, B.E.A., Teich, M.C.: Fundamentals of Photonics. Wiley, New Jersey (2007) 
Selim, R., Pinto, D., Obayya, S.S.A.: Novel fast photonic crystal multiplexer-demultiplexer switches. Opt. Quant. Electron. 42(8), 425-433 (2011)

Sözüer, H.S., Sengün, H.D.: Photonic crystal assisted 90 waveguide bend. Int. J. Mod. Phys. 25, 2167$2182(2011)$

Taflove, A., Hagness, S.C.: Computational Electrodynamics: The Finite-Difference Time-Domain Method. 3rd edn. Artech House Publishers, Norwood (2005)

Taillaert, D., Chong, H., Borel, P., Frandsen, L., DeLa Rue, R., Baets, R.: A compact two-dimensional grating coupler used as a polarization splitter. IEEE Photon. Technol. Lett. 15, 1249-1251 (2003)

Tingchao, H., Yongguang, C., Yabing, D., Yujun, M.: Z-scan determination of third-order nonlinear optical nonlinearity of three azobenzenes doped polymer films. Opt. Commun. 275, 240-244 (2007)

Vigneron, J.P., Lousse, V.: Theory of chirped photonic crystals. Opt. Quant. Electron. 39, 377-385 (2007)

Xiaoqiang, Z., Changshun, W., Yi, Z., Zhang, J.: Nonlinear refraction and photoinduced birefringence in the methyl-red-doped polymer thin film. J. Nonlinear Opt. Phys. Mater. 19, 437-444 (2010)

Yesayan, A., Piche, M.: Grating-assisted coupler used as a narrow bandwidth channel-dropping filter. Opt. Quant. Electron. 36, 1221-1225 (2005)

Yokoi, N., Fujisawa, T., Saitoh, K., Koshiba, M.: Apodized photonic crystal waveguide gratings. Opt. Express 14, 4459-4468 (2006) 